\title{
The Influence of Multi-Touch Interaction on Procedural Training
}

\author{
Sarah Buchanan \\ University of Central Florida \\ Orlando, FL \\ sarahb@cs.ucf.edu
}

\author{
Jared Bott \\ University of Central Florida \\ Orlando, FL \\ jbott@cs.ucf.edu
}

\author{
Joseph J. LaViola Jr. \\ University of Central Florida \\ Orlando, FL \\ jjl@eecs.ucf.edu
}

\begin{abstract}
This paper explores the use of multi-touch interaction in a 3D training environment as a way to enhance learning of sensorimotor skills as well as procedural knowledge. We present a between subjects experiment with 36 participants distributed into 3 groups that use multi-touch interaction, interaction with the physical apparatus, and a control group using basic mouse-based interaction. A post-training test carried out 3 days later evaluated performance in conducting the real world task from memory. Results show that the multitouch interaction and the real world groups had significantly better performance scores than the mouse interaction group, with no significant difference between multi-touch and real world groups. Our results demonstrate that multi-touch interaction trained participants on the task as well as training on the actual equipment, indicating multi-touch interaction is a potential replacement for the physical apparatus when doing procedural training.
\end{abstract}

\section{ACM Classification Keywords}

H.5.2. Information Interfaces and Presentation (HCI): Miscellaneous

\section{Author Keywords}

Multi-touch; surface; procedural training; transfer learning; user study

\section{INTRODUCTION}

Multi-touch Tabletops and Surfaces have been used for a multitude of applications such as visualizing information [17] , inquiry-based learning [14], collaboration [6], neurorehabilitation [1], and strategic simulation training [2]. However, not much exploration has been done in leveraging multitouch interaction for procedural training such as an industrial assembly tasks, mechanical repairs, or medical procedures. Industrial assembly or repairs are usually taught through video documentation, 2D mechanical drawings, or the explanation from an expert. Traditional medical education relies

Permission to make digital or hard copies of all or part of this work for personal or classroom use is granted without fee provided that copies are not made or distributed for profit or commercial advantage and that copies bear this notice and the full citation on the first page. Copyrights for components of this work owned by others than ACM must be honored. Abstracting with credit is permitted. To copy otherwise, or republish, to post on servers or to redistribute to lists, requires prior specific permission and/or a fee. Request permissions from Permissions@ acm.org.

ITS '15, November 15 - 18, 2015, Funchal, Portugal

Copyright is held by the owner/author(s). Publication rights licensed to ACM. ACM 978-1-4503-3899-8/15/11: \$15.00.

DOI: http://dx.doi.org/10.1145/2817721.2817740

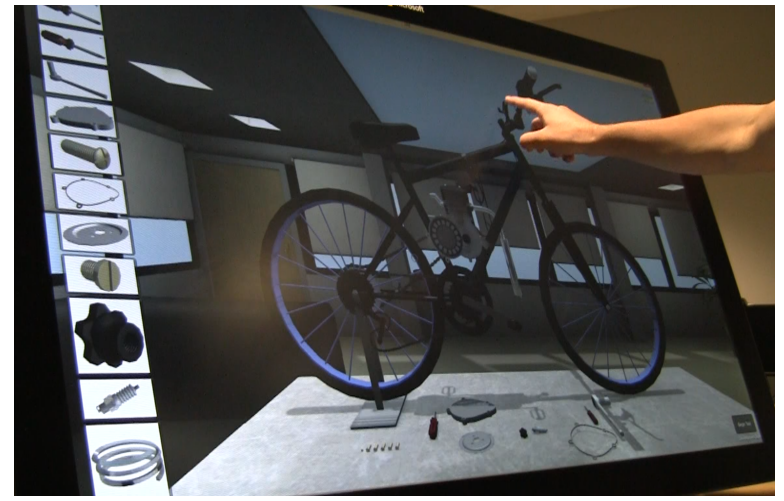

Figure 1: The motorized bicycle model in the virtual environment with the tools and parts on the table or in the tool bar for easy access.

heavily on lectures for factual knowledge, observing professionals and treating patients under supervision. However, enactive learning and interfaces have been shown to be more effective than passive learning methods [10]. Enactive interfaces have been shown to be especially effective when optimizing affordances, or possible actions available to someone using the interface [16]. We aim to leverage the combined affordances of a multi-touch surface and a virtual environment to provide an effective replacement for their physical counterparts as a way to reduce cost and save time.

Our goal is to evaluate the training benefits of a multi-touch training system that provides realistic affordances, by way of physical multi-touch gestures in a virtual environment. This paper focuses on the use of a multi-touch surface for learning a procedural task, specifically learning a repair and assembly task, and the transfer of knowledge to the real world task. Mechanical repair tasks, as well as medical procedures, are complex tasks which involve the knowledge of specific procedures, the location of parts, the interactions between parts, and the use of tools. We demonstrate realistic physical gestures and interaction techniques that aid in learning by creating realistic constraints that would be applicable to a variety of procedural tasks.

Specifically, we address the following research question: How does multi-touch interaction affect the learning of a procedural assembly task? To address this question, this paper presents a study evaluating the knowledge transfer acquired 
with multi-touch interaction technology compared to standard training methods. We compared multi-touch interaction to standard 2D mouse interaction and to actual physical training.

\section{RELATED WORK}

\section{Multi-touch Interaction}

There has been much work in interactive tabletops in education; for experiential learning [5], to enhance collaborative learning [13] or encourage inquiry-based learning [14], [15]. The Flow of Electrons [5] provides an augmented workspace for learning physical computing experientially. By allowing users to place electronics components on the surface and experiment with wiring and the outcomes, it allows users to make mistakes without fear of breaking anything. It bridges the gap between digital information and actual hardware components. Similarly, in [13] the affordances of study materials on the tabletop versus paper allowed for more playfulness and experimentation since the notes or drawings created were not permanent and were easily edited. In G-nome Surfer, a tabletop interface for learning genomics, users demonstrated a higher degree of cooperative behavior and higher test scores on tabletops compared to traditional GUI setups in genomics study. The ability for users to easily and immediately experiment and practice without the fear of making permanent mistakes is something that can benefit training applications as well, since users may be intimidated by expensive equipment or dangerous medical procedures.

Multi-touch interfaces have not been explored as much in the context of training. OrMiS [2], a tabletop interface for simulation-based training, where military officers use a mapbased tool to carry out strategic maneuvers and combat, enabling large-scale training exercises without the cost of field deployment. OrMiS is designed to replace traditional PCbased simulation tools while improving ease of learning and better facilitating collaborative work. OrMis focuses on 2D map-based strategic tasks whereas we focus on training on a physical apparatus in a 3D environment using realistic gestures. For medical training SimMed implements a 3D simulation in an augmented tabletop environment to teach medical procedural skills for diagnosis and treatment [19]. They observed high levels of immersion and positive social aspects, as well as observations that suggest a significant learning effect. Their training was very open-ended in order to explore the diagnostic techniques trainees chose, whereas we are focused on training a specific step-by-step procedure. There are no other studies, that we are aware of, which evaluate the efficiency and effectiveness of procedural training using multi-touch interactive surfaces.

\section{VR and AR Procedural Learning}

Although there hasn't been much work with multi-touch systems in the areas of maintenance and medical training, there has been much work with VR and AR systems $[8,9,11,12]$. There have been many studies evaluating the effectiveness of VR and AR compared to traditional methods, reporting in some cases that these methods perform as well as traditional hands-on methods. For instance, Ganier et al. compared tank

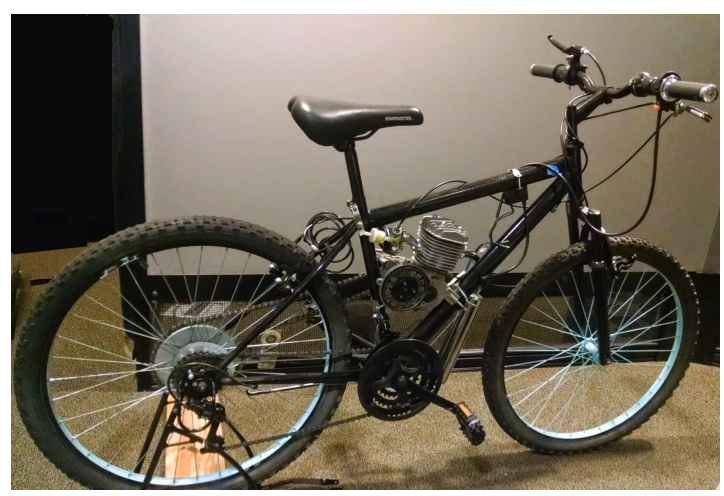

(a)

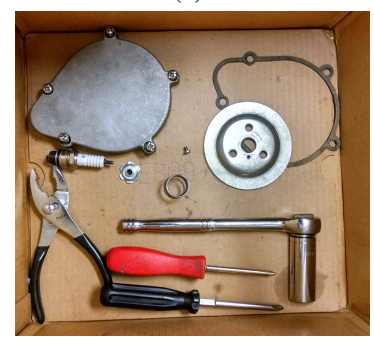

(b)

Figure 2: The physical motorized bicycle apparatus (2a) with the accompanying tools and parts (2b).

maintenance procedure performance after conventional training with a real tank and those trained in a virtual environment. Both training groups were also compared to a no-training control group who carried out the procedure using only job instructions [7]. No differences were found for the testing task completion times for the test groups, but significant differences were found between both test groups and the control group. Indicating that procedural knowledge learned in a virtual environment is transferred to real-world performance. Another study evaluated different Virtual Reality interaction technologies in learning an industrial maintenance task [18]. The four different interaction technologies were basic mouse, 2D mocap, 3D mocap, and a haptic device. They found negligible impact on the learning of assembly task when the focus is on transfer of procedural knowledge rather than the transfer of sensorimotor skills. In addition, users that trained with mouse and 2D mocap took significantly less time training.

Henderson and Feiner evaluate an augmented reality (AR) user interface designed to assist users in the psychomotor phase of procedural tasks [9]. Their user study showed participants completed the psychomotor aspects of the assembly task significantly faster and with greater accuracy than when using assistance on a stationary LCD screen. Another study examined VR and AR techniques compared with classic training techniques. The VR and AR methods showed no significant differences over control methods. In addition, they found AR training for industrial maintenance and assembly (IMA) tasks can reduce the number of unsolved errors [7]. One of the main differences with these AR and VR studies is they they make use of 3D spatial interfaces [3] while 


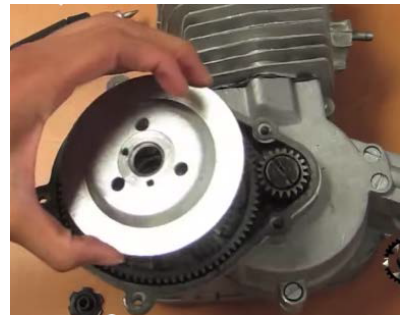

(a) Assemble Clutch

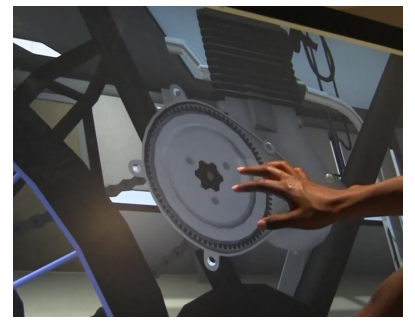

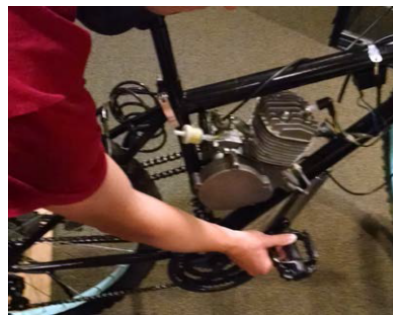

(b) Test and adjust clutch

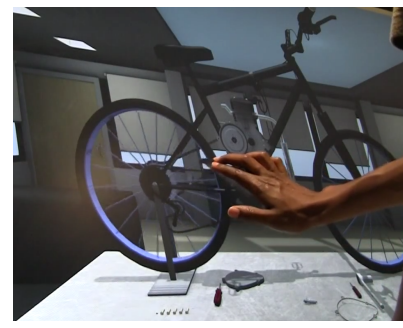

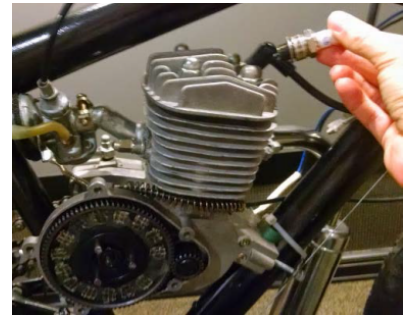

(c) Install spark plug

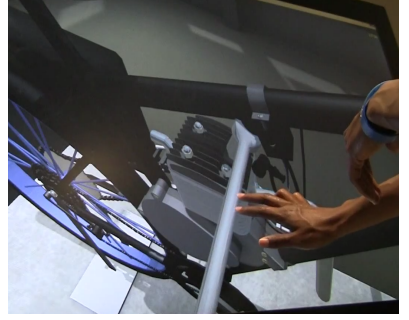

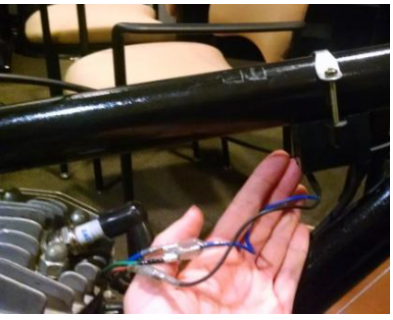

(d) Configure wiring

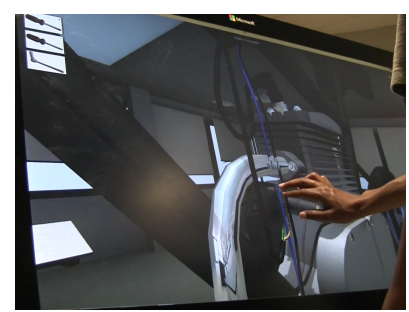

Figure 3: The four different sub-tasks (3a-3d) shown on the physical bicycle apparatus (top), and in the virtual environment (bottom).

we focus on 2D multi-touch gestures. We aim to expand upon these results in virtual procedural training by exploring whether multi-touch interaction, which leverages realistic gestures, can perform as well as training on the real equipment.

\section{TRAINING SYSTEMS AND TASK}

The selected experimental task consisted of assembling and testing part of a 2 -stroke bicycle-mounted engine (shown in Figure 2). The apparatus and task were selected based upon a number of constraints: (1) the task should use tools, parts and gestures that would be applicable to industrial, defense, or medical tasks, (2) the apparatus should be able to fit into our lab, (3) the task should be safe for all participants to perform, (4) the task should be significantly complex and non-obvious that a layman could not complete it without prior training. These constraints led us to repairing a 2-stroke bicycle engine where participants could assemble, test and adjust the clutch and other parts.

The overall task consisted of 22 steps grouped into 4 sub-tasks (shown in Figure 3): (1) clutch assembly, (2) clutch functionality testing and adjustments, (3) spark plug installation, and (4) wire configuration. Some examples of the operations participants had to perform are: tightening the clutch wheel nut to adjust the clutch plate, tightening screws with a screwdriver, installing the spark plug with a socket wrench, and plugging in wires. The task also required verifying the correct clutch plate tightness, where the engine needed to be disengaged with the clutch in and engaged with the clutch out. If either of these conditions are not true the clutch plate should be looser or tighter respectively. In total, participants had to manipulate roughly 25 parts, and use three different types of tools (shown in Figure $2 b$ ).

\section{Motorized Bicycle}

The physical apparatus was a motorized bicycle mounted on a small stand to allow pedaling the bike freely in place for testing purposes. The participants that trained on the physical bicycle had the advantage of experiencing the affordances provided by each of the tools and parts involved in the task. For instance, understanding the correct tightness of the clutch plate is an important step in the process. This can be experienced by tightening the nut about $80 \%$ of the way and making sure the clutch plate still has about $1 / 8$ " of movement. As described above, correct clutch plate tightness can be verified by pedaling the bike with both the clutch in and the clutch out. The user can verify the engine is disengaged visually by looking at the gears and observing the relative easiness of pedaling the bike. With the engine engaged, the user can feel that the bike is more difficult to pedal and hear the engine making a chugging sound. The ability to examine the parts and bike apparatus from any viewpoint or zoom level is also an advantage the physical model provides. As described above, the physical bicycle provides feedback in the form of opposing forces and aural and visual cues.

\section{Virtual Model and Multi-touch Interaction}

The virtual environment contained a detailed, working model of the bicycle, engine, components and tools involved (shown in Figure 1). A tool bar was also provided so that if a user was zoomed into a certain area of the bike they wouldn't have to zoom out to select a part from the table. Users could select the tool or part needed from the tool bar and it would hover next to the button. Each corresponding step in the task was replicated in the virtual model and required interaction. We ensured both the multi-touch and mouse interaction required the same number of steps to complete the task.

Previous work on user-defined multi-touch gestures for 3D objects influenced our multi-touch interaction model [4]. 
Based on this work, all objects, except for small screws, required two or more fingers to manipulate in order to make the interaction more realistic. The gestures incorporated were double tap, translate, rotate, pressure, grasp, grip and hold. Double tap zoomed in and out of the 5 main viewpoints of the bike. Translate was used to move parts and tools around. Rotate was used on the clutch plate to align it, and on the screwdrivers. Pressure was used to apply pressure when installing the spring and to attach the socket wrench to the spark plug. Pressure information was interpreted based off touch point size provided by the display device. Grasp was used on the clutch lever; multiple fingers had to push down on the lever while the thumb stayed on the handle grip. Grip was used on the socket wrench handle to grip it and rotate it around. Hold was used in 2-handed gestures where gravity or other forces would take over. For instance, if the user let go of the clutch plate before securing the clutch wheel nut with the other hand the plate would detach due to the force of the spring and fall to the table.

Where possible we aimed to replicate the affordances provided by the real model in the virtual model and multi-touch gestures. For example, the steps in the real task and the corresponding steps replicated in the virtual model follows and are shown in Figure 4. In the real model installing the clutch plate requires: (1) pressing and rotating the spring over the center bolt until it stays, (2) attaching the clutch plate by rotating it so it aligns with the three pegs, and then (3) holding and pressing the clutch plate over the spring while screwing on the clutch wheel nut. The corresponding multi-touch interaction required was identical, where the gestures required were: (1) translate and pressure rotate the spring, (2) translate, rotate and hold the clutch plate, (3) 2-handed interaction where one hand holds the clutch plate while the other hand translates and rotates the clutch wheel nut.

In the above example, even though the main gesture motions were identical, some affordances were not replicated due to the constraints of the interface. For instance, in the physical model you have to rotate and press on the spring until it clicks onto the center bolt, but sometimes this is tricky and can be hard understand how it feels when the spring attaches. Whereas in the multi-touch model, the user only needs to press and rotate 180 degrees and it will automatically connect. On the other hand, rotating and aligning the plate was more difficult in the multi-touch model since the user is required to exactly match up the plate with the three pegs and then press. In the physical model the same precision is not required. For step (3), the physical model's spring pushed back against the clutch plate reminding users to apply pressure to keep it in place. In the multi-touch environment, users had no such feedback and may forget that pressure is required to prevent the plate from detaching until the nut is secure.

We emphasized the model fidelity and interaction details even further to articulate any sensorimotor skills that wouldn't be found in the user manual alone. As for interaction details, the number of rotations required to rotate the clutch wheel nut, the screws with the screwdrivers, and the spark plug with socket wrench were the same for the actual bicycle appara-
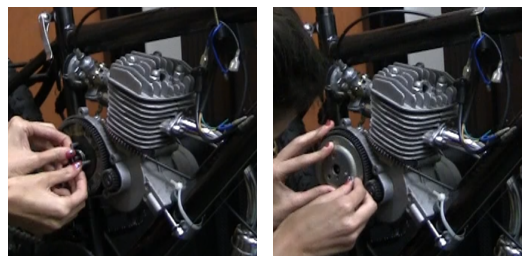

(a) Pressing and ro- (b) Pressing and aligntating the spring over ing the clutch plate the center bolt until it over the spring and clicks

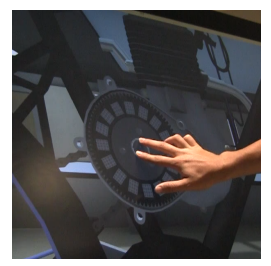
three pegs

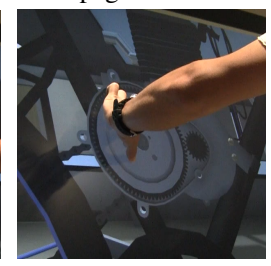

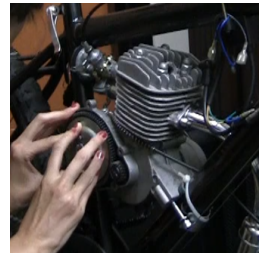
clutch wheel nut over the clutch plate to the appropriate tightness

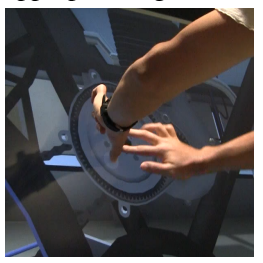

(c) Tightening the

Figure 4: The three steps in assembling the clutch $(4 a-4 c)$ shown on the actual bicycle apparatus (top), and in the virtual environment (bottom).

tus and the virtual model. As for model fidelity, in order to get the clutch plate to the correct tightness, participants were directed to tighten the clutch wheel nut approximately $80 \%$ of the way and then perform the clutch testing procedure to verify it was working correctly. In the virtual model, $80 \%$ tightness also mapped to $1 / 8$ " of clutch plate movement so users could press on the clutch plate and see how much it depressed. We also indicated that it was harder to pedal the bicycle with the engine engaged by requiring 2 fingers and pressure to pedal when engaged and 1 finger versus no pressure threshold when disengaged.

\section{Virtual Model and Mouse Interaction}

The mouse interaction was intended as a control training group, providing only basic click and drag interaction. We implemented a simplified mouse condition over an optimized mouse condition to demonstrate that it's not only the number of steps the user completes, but the level of interaction that affects learning. We ensured both the multi-touch and mouse interaction required the same number of steps. With the mouse the user would drag the part or tool to the desired position and then when close enough it would snap to place. To perform rotations the user would click again and the part or tool would animate as it rotated into place. If a mistake was made, the user could click to uninstall and then move the part away. Since rotations only required one click to animate they could be completed much quicker than in the multi-touch interface where a user would have to perform multiple rotation gestures to install the part completely.

\section{USER STUDY}

We conducted a user study to explore the effectiveness of gesture-based multi-touch training. The study examined three different methods of training how to repair a 2-stroke bicycle engine. The three methods were using the actual equipment, 
using a virtual model with gesture based interaction, and using a virtual model with a mouse. All groups trained with the same paper manual and returned three days later for a posttraining test. During the post-test participants were asked to perform the task on the actual equipment as quickly as possible from memory and were graded on correct steps and timed. Our hypotheses were:

H1 The participants trained using the gesture interface will score as well on the post-test as the participants trained with the actual equipment.

$\mathrm{H} 2$ The participants trained using the gesture interface will score better on the post-test than the participants trained using the mouse interface.

H3 Due to discrepancies between manipulating the actual components and the virtual model, the participants trained using the gesture interface will perform the post-test slower than the participants trained with the actual equipment.

H4 Since the gesture participants may have maintained some muscle memory of the task, participants trained using the gesture interface will perform the post-test faster than the participants trained using the mouse interface.

H5 The training time for the mouse participants will be faster than the bicycle and multi-touch groups since they don't have to go through the longer physical gestures.

\section{Participants and Apparatus}

There were 36 participants (13 female, 23 male) aged 18 to $37(\bar{x}=23.6, \sigma=4.58)$ randomly distributed into the three groups. The participants were recruited from a University setting from a wide variety of majors including Speech Pathology, Biology, Marketing, Business, Information Technology, and more. Participants rated their mechanical experience on a Likert scale from 1 to $7,(\bar{x}=3.06, \sigma=2.04)$, and none of the participants had worked on a similar engine previously. Training was either conducted on the computer via a 55-inch Microsoft Perceptive Pixel multi-touch screen, or on the actual bicycle and engine. Post-tests were performed on the physical bicycle.

\section{Study Design}

The experiment follows a between-subjects design with 36 participants randomly divided into 3 experimental groups (each participant was assigned to a group in alternating order). Two groups trained in a virtual environment, one using multi-touch gestures or the other using mouse input. The third group was trained on the actual bicycle.

Participants were timed during training and testing and scored on the test. Participants were also asked to rate the following statements on a Likert scale from 1 (strongly disagree) to 7 (strongly agree), both post-training and post-testing:

Post-Training questions:

Q1 I feel prepared to complete the repair task after completing the training

Q2 I thoroughly understand the concepts that I learned during the training

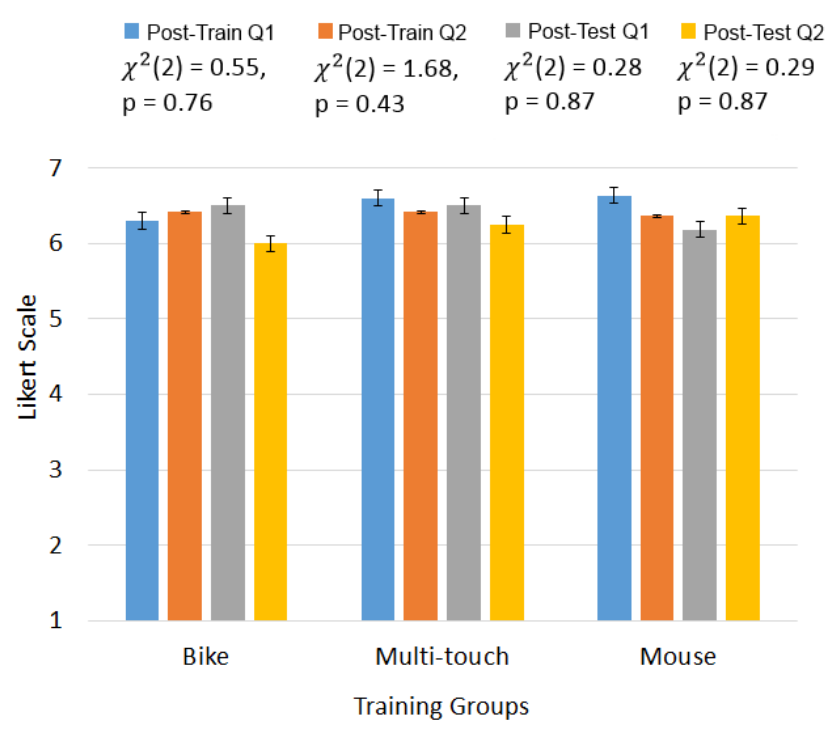

Figure 5: Average user Likert ratings on the preparedness and understanding questions asked after training and after testing.

\section{Post-Testing questions:}

Q1 I was sufficiently prepared to complete the repair task upon arrival today

Q2 I thoroughly understood the concepts that I learned during the training

\section{Procedure}

Testing occurred exactly 3 days after training to avoid shortterm memory effects. For training, all groups followed the same protocol. First, participants were presented with the training apparatus (whether it be the computer or bicycle) and the paper manual. Second, they were familiarized either with the tools or the interaction model. Second, participants were asked to follow along with the paper manual as they conducted the task. They were told they could complete the training as many times as they wanted, so long as they could learn the task and describe the process back to the proctor correctly.

Three days later, participants returned to perform the task on the real bicycle engine. They were asked to perform the task correctly from memory without the user manual. They were scored on the steps shown in Table 1. One point was given for each step completed correctly and performed in the correct order, for a total of 22 points. If they skipped a step then later remembered and backtracked to complete it, they received a $1 / 2$ point.

\section{RESULTS}

\section{Training}

\section{Training Time}

The participants could go through the training as many times as they wanted to learn the task and describe the process back to the proctor, the average total training time by group is 


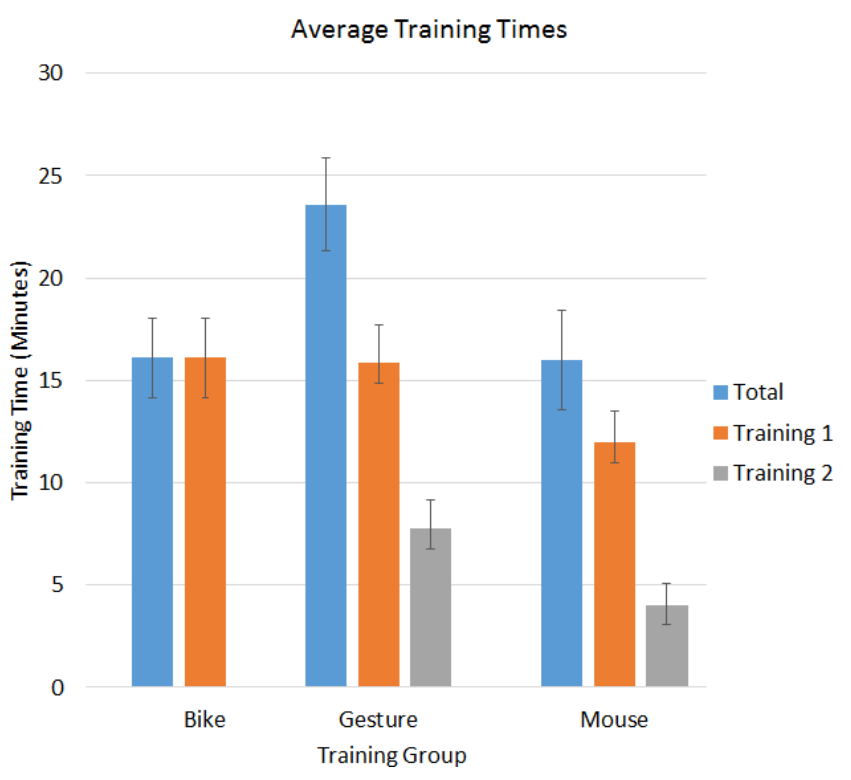

Figure 6: Average training times by training group. Participants could go through the training multiple times, however the participants in the physical bike group all opted to only perform the training once.

shown in Figure 6. We found that all of the participants in the Bike group only went through the process once, whereas all of the participants in the Multi-touch and Mouse groups went through the training twice. The bike trainees would have to wait on the proctor to dismantle the training model which may have discouraged them from going through it twice. Once the virtual users had learned the interface, they were able to quickly go through the training a second time. The virtual medium affords quickly repeating the training scenario as compared to the physical training apparatus.

\begin{tabular}{|ll|rl|}
\hline & Steps & \multicolumn{2}{|c|}{ Steps } \\
\hline 1 & Clutch lever & 11 & Testing - clutch \\
2 & Clutch button & 12 & down to adjust \\
3 & Spring & 13 & Flathead screw \\
4 & Clutch plate & 14 & Gasket screwdriver \\
5 & Clutch wheel nut & 15 & Gear case cover \\
6 & Begin testing & 16 & 5 screws \\
7 & Testing - pedal bicycle & 17 & Phillips Screwdriver \\
8 & Testing - clutch down, & 18 & Spark plug \\
& engine disengaged & 19 & Socket wrench \\
9 & Testing - clutch up, & 20 & CDI cap \\
& engine engaged & 21 & Connected wiring \\
10 & Testing - diagnose & 22 & Wiring correct \\
& correct adjustment & & \\
\hline
\end{tabular}

Table 1: Steps in the procedure that participants were scored on during the post-test.

An independent samples t-test did indicate the multi-touch group spent significantly more time than the bike group $\left(t_{22}=\right.$ $-2.42, p<0.025)$ and the mouse group $\left(t_{22}=-2.96, p<0.007\right)$ on the first training session. However, the bike and mouse

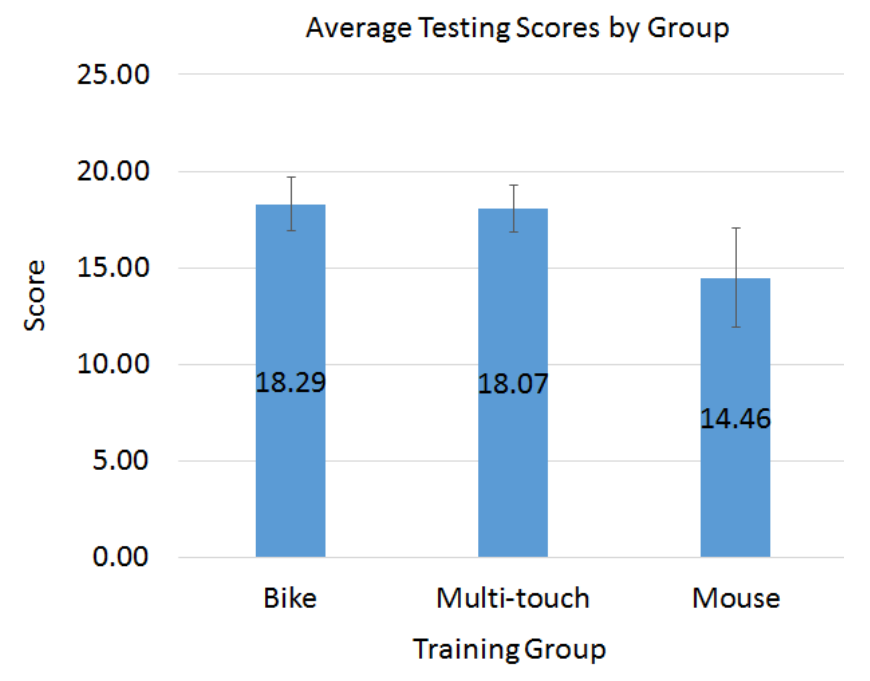

Figure 7: Average testing scores by training group.

groups training times were not significantly different $\left(t_{22}=\right.$ $0.623, p=0.540)$. This result demonstrates that multi-touch participants had to perform detailed, realistic interactions and may have also spent extra time adjusting to new gestures.

\section{Questionnaire}

We performed a Kruskal-Wallis test (results are shown in Figure 5) which showed that there were no significant differences between the different groups for their responses to each of the 4 Likert questions (presented in the Participants and Apparatus section). These results indicate that participants from all groups felt that they were prepared after the training, and still felt that they were prepared after performing the actual posttest.

\section{Testing}

\section{Testing Scores}

When the participants returned for testing, they were asked to perform the task on the real bicycle and engine from memory. They were scored on number of steps completed correctly and timed. The average scores by group are shown in Figure 7. The scores were normally distributed for the Bike, Gesture, and Mouse groups as assessed by Shapiro-Wilk's test $(\mathrm{p}>.05)$. A one-way between-subjects analysis of variance (ANOVA) was run to compare the effect of the training method on the testing score. The main effect that training method had on the dependent variable, testing score, was found to be significant $(\mathrm{F}(2,33)=5.36, \mathrm{p}<0.010)$. Independent samples t-tests indicated the gesture group scored significantly better than the mouse group $\left(t_{22}=2.50, p<0.025\right)$ and the bike group also scored significantly better than the mouse group $\left(t_{22}=2.58, p<0.020\right)$. However, the bike and gesture groups scores were not significantly different $\left(t_{22}=0.240, p=0.812\right)$. This result demonstrates that participants that trained with Multi-touch performed as well as those trained on the actual apparatus and better than those trained on the Mouse. 


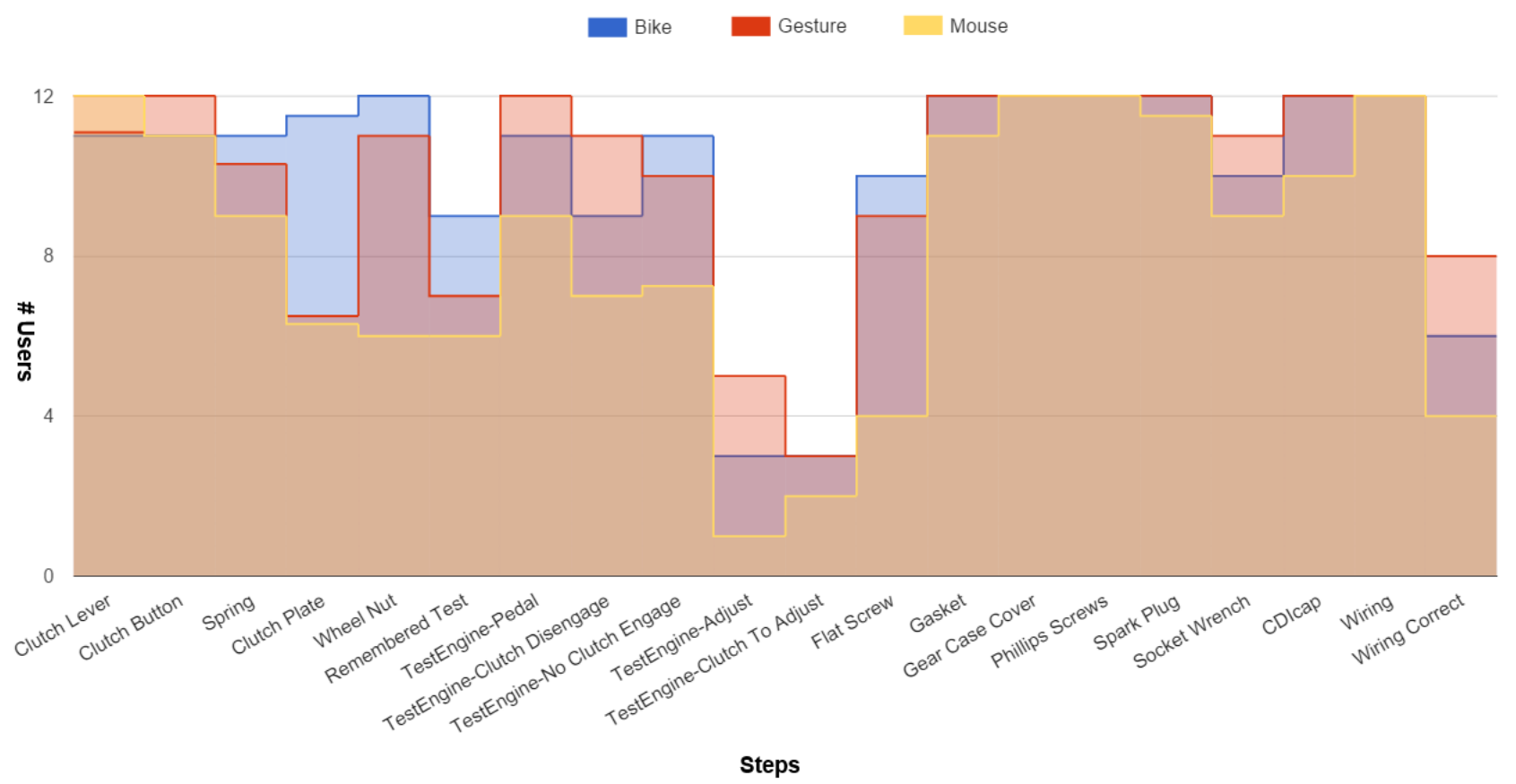

Figure 8: The number of users that completed each testing step correctly for each training group; the bike group in blue, the gesture group in red, and the mouse group in yellow. This graph also demonstrates on which steps users made the most errors by showing where the deficits are. For example, for the flat screw step only 4 of the mouse users performed the step correctly, whereas 9 gesture users and 10 bike users performed it correctly.

\section{Testing Errors}

Figure 8 shows each step of the testing process and how many users got the step correct, separated by testing groups. The errors occurred when a user forgot or skipped a step. If a user remembered to do a step, there was never a case where the user didn't know how to operate the tool or part in that step, but instead users completely forgot the process altogether.

\section{Testing Scores}

The testing times were also measured (shown in 9) and found to be normally distributed for the Bike, Gesture, and Mouse groups, as assessed by Shapiro-Wilk's test ( $\mathrm{p}>.05)$. A oneway between-subjects analysis of variance (ANOVA) was run to compare the effect of the training method on the testing score. The main effect that training method had on the dependent variable, testing score, was not significant $(\mathrm{F}(2,33)=$ $1.64, \mathrm{p}=0.209)$.

\section{Discussion}

The performance results (shown in Figure 7) demonstrate that participants trained with multi-touch interface scored as well as those trained on the actual apparatus and better than those trained on the mouse interface, confirming Hypotheses $\mathrm{H} 1$ and H2. However, the post-test times were not statistically significant and do not confirm Hypotheses H3 and H4. This could be due to a few reasons. It's possible incorrect steps could still lead to the same testing time as a test that was completed without errors. In addition, the inexperience of some participants using tools, causing them to be more cautious than others, may have lead to higher variance within

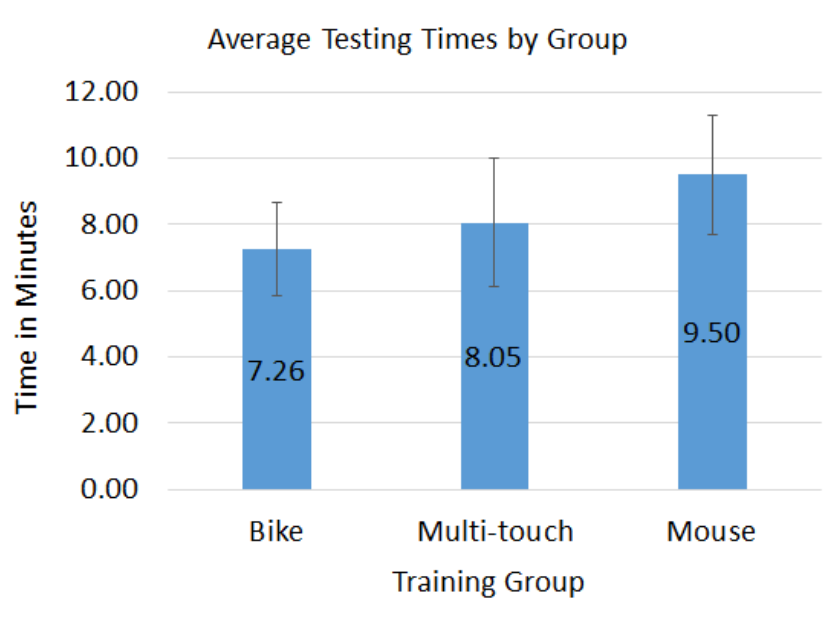

Figure 9: The average testing times by training group.

groups. We believe that a few factors led to the multi-touch group scoring as well as the bike group. First, the affordances of the multi-touch model were closely mapped to the affordances of the real model, allowing multi-touch users to perform nearly the same physical gestures in the simulation as they would in real life. Secondly, strict constraints used in the multi-touch training (discussed in the Virtual Model and Multi-touch Interaction section) demanded more concentration allowing better memory of the steps as well as the physical motions required. Thirdly, users felt that repeating 
the training would be quick and easy since they could simply restart the environment to start again with the dismantled model versus undoing each step performed on the physical model.

Hypothesis H5 was partially confirmed, the Mouse group trained faster than the Multi-touch group but not the Bike group. We believe the gesture group had longer training times since there was a gesture learning curve and mistakes were made more often during training due some strict constraints. It could be argued that the multi-touch group performed better than the mouse group since they spent more time training. However, the majority of this time was spent learning, adjusting and executing gestures, not in additional training repetitions. Since the number of steps were identical and the virtual model was the same between the mouse and multitouch groups, we believe the better multi-touch performance was due to the similarities of the multi-touch model affordances and physical gestures with the actual bicycle model and physical movements. Although the multi-touch training was not as efficient, time-wise, as the mouse training it was more effective.

The multi-touch interface provides the ability for users to manipulate tools and parts and perform realistic physical gestures as they would in a real-world procedure. Although our gesture set was somewhat small (7 gestures), it is still possible to achieve realistic actions; the gestures become more realistic when tied to physics forces and constraints. It is important for the physical motions and constraints within the procedural steps to be replicated where possible. For instance, consider a task in real life where one hand is required to hold a part in place while the other attaches it. The corresponding multitouch interaction should also require a 2-handed gesture instead of objects snapping into to place or ignoring gravity. In some instances the stricter constraints in the multi-touch interaction may have led to more mistakes by users in training, but may have forced them to learn the steps better since they had to use more precise gestures.

For instance, a more complex 2-handed gesture was used for steps 4 and 5 (from Table 1) which required holding the clutch plate while tightening with the clutch wheel nut. As shown in Figure 8, more multi-touch users remembered the wheel nut step versus mouse users, potentially due to this increased amount of interaction. On the other hand it is interesting that approximately the same number of mouse and multi-touch users missed the clutch plate step. Some virtual users said this was because the shininess and/or shape of the plate in real life was not reflected in the virtual model and was hard to recognize.

In order to maintain the feeling of holding and manipulating the parts directly, or direct manipulation, we chose the viewpoints in the virtual environment carefully. A perceptual disconnect can occur when an object moves away from a user's touch and the object is no longer underneath the user's hand. This is particularly apparent when translating objects along multiple axes.
On a multi-touch interface, the user gets force feedback from the surface, however they don't get full 3DOF positional force nor any rotational force feedback. We chose to simulate applying force with pressure and use of more fingers. Pressure and the number of fingers required are good indicators of force but don't translate exactly to force in real life. For instance, attaching the spring to the bolt in the physical model requires pressing hard while rotating. In the multitouch model users need to rotate 180 degrees and meet a pressure threshold. During the post-test, the multi-touch users expressed confusion as to why the spring wasn't attaching as easily as in the training.

For force feedback, the user could also be required to apply an opposing force in the form of more fingers and more pressure. For instance, the physical model's spring pushed back against the clutch plate reminding users to apply pressure to keep it in place. In the multi-touch environment, users had no such feedback and may forget that pressure is required to prevent the plate from detaching until the nut is secure. By requiring the user, in this instance, to apply pressure as an opposing force, they may learn the existence of force feedback in that action. Pressure could be improved upon by training users on how pressure is measured on a touch screen, calibrating pressure on a per user basis, or by providing visual and aural cues. Since forces cannot be felt by the user, visual and aural cues must compensate for that lost sense of force.

The realistic multi-touch gestures were tightly linked with physics in the virtual environment, procedural constraints, simulating forces, and carefully chosen viewpoints in order to create a convincing simulation of the physical actions within the maintenance procedure. Keeping in mind these considerations, multi-touch can provide a realistic and effective training environment for procedural tasks.

\section{FUTURE WORK}

In the future we would like to simulate more of the affordances provided by the physical model. For instance, we could add more pressure requirements to try to increase realism in the multi-touch condition. We could also provide aural cues to replace haptics or add tactile buzzing on the interaction surface to support better tactile feedback. In future experiments, examining how increasing the training time with each method affects learning would be interesting. Since we had the participants use a paper training manual we would like to incorporate training instructions and guidance into the virtual environment. We would also like to do a similar study comparing multi-touch gestures to Virtual Reality, full body gestures, and training on physical systems using Augmented Reality potentially on a more complex procedural task.

\section{CONCLUSION}

In this paper we presented the use of realistic multi-touch interaction in a 3D training environment as a way to enhance learning of sensorimotor skills a well as procedural knowledge. We conducted a between subjects experiment with 36 participants distributed into 3 groups in order to evaluate the effectiveness of multi-touch training. One group used multitouch interaction in the 3D training environment, the sec- 
ond used basic mouse-based interaction, and the third trained on the real equipment. A post-training test carried out 3 days later evaluated performance in conducting the real task from memory. Results show that the multi-touch interaction and the real task groups had significantly better performance scores than the mouse interaction group, with no significant difference between multi-touch and real task groups. We demonstrated that multi-touch interaction trains participants on the task as well as training on the actual equipment, suggesting multi-touch interaction is a worthwhile training tool for procedural knowledge that requires sensorimotor skills.

\section{ACKNOWLEDGMENTS}

This work is supported in part by NSF CAREER award IIS0845921 and NSF award CCF-1012056. Also, we would like to thank the members of ISUE lab for their support and the anonymous reviewers for their useful comments and feedback.

\section{REFERENCES}

1. Mirjam Augstein, Thomas Neumayr, and Irene Schacherl-Hofer. 2014. The Usability of a Tabletop Application for Neuro-Rehabilitation from Therapists' Point of View. In Proceedings of the Ninth ACM International Conference on Interactive Tabletops and Surfaces (ITS '14). ACM, New York, NY, USA, 239-248.

2. Christophe Bortolaso, Matthew Oskamp, T.C. Nicholas Graham, and Doug Brown. 2013. OrMiS: A Tabletop Interface for Simulation-based Training. In Proceedings of the 2013 ACM International Conference on Interactive Tabletops and Surfaces (ITS '13). ACM, New York, NY, USA, 145-154.

3. Doug A Bowman, Ernst Kruijff, Joseph J LaViola Jr, and Ivan Poupyrev. 2004. 3D user interfaces: theory and practice. Addison-Wesley.

4. Sarah Buchanan, Bourke Floyd, Will Holderness, and Joseph J LaViola. 2013. Towards user-defined multi-touch gestures for 3D objects. In Proceedings of the 2013 ACM international conference on Interactive tabletops and surfaces. ACM, 231-240.

5. Bettina Conradi, Verena Lerch, Martin Hommer, Robert Kowalski, Ioanna Vletsou, and Heinrich Hussmann. 2011. Flow of electrons: an augmented workspace for learning physical computing experientially. In Proceedings of the ACM International Conference on Interactive Tabletops and Surfaces. ACM, 182-191.

6. Veronika Domova, Elina Vartiainen, and Marcus Englund. 2014. Designing a Remote Video Collaboration System for Industrial Settings. In Proceedings of the Ninth ACM International Conference on Interactive Tabletops and Surfaces (ITS '14). ACM, New York, NY, USA, 229-238.

7. Franck Ganier, Charlotte Hoareau, and Jacques Tisseau. 2014. Evaluation of procedural learning transfer from a virtual environment to a real situation: a case study on tank maintenance training. Ergonomics 57, 6 (2014), 828-843.

8. Florian Gosselin, Sylvain Bouchigny, Christine Mgard, Farid Taha, Pascal Delcampe, and Cdric dHauthuille. 2013. Haptic systems for training sensorimotor skills: A use case in surgery. Robotics and Autonomous Systems 61, 4 (2013), 380 - 389. Models and Technologies for Multi-modal Skill Training.

9. S.J. Henderson and Steven K. Feiner. 2011. Augmented reality in the psychomotor phase of a procedural task. In Mixed and Augmented Reality (ISMAR), 2011 10th IEEE International Symposium on. 191-200.

10. DL Holton. 2010. Constructivism+ embodied cognition $=$ enactivism: theoretical and practical implications for conceptual change. Recuperado a partir de http://bit. ly/gVAOOu (2010).

11. Sung Gu Kang, Byung Ju Ryu, Kyung Sook Yang, Young Hwii Ko, Seok Cho, Seok Ho Kang, Vipul R. Patel, and Jun Cheon. 2015. An Effective Repetitive Training Schedule to Achieve Skill Proficiency Using a Novel Robotic Virtual Reality Simulator. Journal of Surgical Education 72, 3 (2015), 369 - 376.

12. M. Kolesnikov, M. Zefran, A.D. Steinberg, and P.G. Bashook. 2009. PerioSim: Haptic virtual reality simulator for sensorimotor skill acquisition in dentistry. In Robotics and Automation, 2009. ICRA '09. IEEE International Conference on. 689-694.

13. Anne Marie Piper and James D Hollan. 2009. Tabletop displays for small group study: affordances of paper and digital materials. In Proceedings of the SIGCHI Conference on Human Factors in Computing Systems. ACM, 1227-1236.

14. Bertrand Schneider. 2012. Designing Tabletop Activities for Inquiry-based Learning: Lessons from Phylogenetics, Neuroscience and Logistics. In Proceedings of the 2012 ACM International Conference on Interactive Tabletops and Surfaces (ITS '12). ACM, New York, NY, USA, 289-294.

15. Orit Shaer, Megan Strait, Consuelo Valdes, Taili Feng, Michael Lintz, and Heidi Wang. 2011. Enhancing genomic learning through tabletop interaction. In Proceedings of the SIGCHI Conference on Human Factors in Computing Systems. ACM, 2817-2826.

16. ThomasA. Stoffregen, BenotG. Bardy, and Bruno Mantel. 2006. Affordances in the design of enactive systems. Virtual Reality 10, 1 (2006), 4-10.

17. Nicole Sultanum, Sowmya Somanath, Ehud Sharlin, and Mario Costa Sousa. 2011. 'Point It, Split It, Peel It, View It": Techniques for Interactive Reservoir Visualization on Tabletops. In Proceedings of the ACM International Conference on Interactive Tabletops and Surfaces (ITS '11). ACM, New York, NY, USA, 192-201. 
18. Yaiza Vélaz, Jorge Rodríguez Arce, Teresa Gutiérrez, Alberto Lozano-Rodero, and Angel Suescun. 2014. The Influence of Interaction Technology on the Learning of Assembly Tasks Using Virtual Reality. Journal of Computing and Information Science in Engineering 14, 4 (2014), 041007.
19. Ulrich von Zadow, Sandra Buron, Tina Harms, Florian Behringer, Kai Sostmann, and Raimund Dachselt. 2013. Simmed: combining simulation and interactive tabletops for medical education. In Proceedings of the SIGCHI Conference on Human Factors in Computing Systems. ACM, 1469-1478. 